\title{
Clinical Profile and Short-Term Outcome of Children With SARS-CoV-2 Related Multisystem Inflammatory Syndrome (MIS-C) Treated With Pulse Methylprednisolone
}

\author{
Sheeja Sugunan, S Bindusha, S Geetha, HR Niyas, A Santhosh Kumar \\ From Department of Pediatrics, SAT Hospital, Government Medical College, Thiruvananthapuram, Kerala.
}

Correspondence to: Dr Sheeja Sugunan, Associate Professor, Department of Pediatrics, SAT hospital, Government Medical College Thiruvananthapuram, Kerala.sheejavimalk@gmail.com Received: February 07, 2021; Initial review: February 25, 2021; Accepted: April 19, 2021.
Objective: To study the clinical profile and outcome of children with MIS-C treated with methylprednisolone pulse therapy and /or intravenous immunoglobulin (IVIG). Method: This prospective observational study included children satisfying CDC MIS-C criteria admitted from September to November, 2020. Primary outcome was persistence of fever beyond 36 hours after start of immunomodulation therapy. Secondary outcomes included duration of ICU stay, mortality, need for repeat immunomodulation, time to normalization of CRP and persistence of coronary abnormalities at 2 weeks. Results: Study population included 32 patients with MIS-C with median (IQR) age of 7.5 (5-9.5) years. The proportion of children with gastrointestinal symptoms was $27(84 \%)$, cardiac was $29(91 \%)$ and coronary artery dilatation was $11(34 \%)$. Pulse methylprednisolone and intravenous immunoglobulin were used as first line therapy in $26(81 \%)$, and $6(19 \%)$ patients, respec-tively. Treatment failure was observed in $2 / 26$ patients in methylprednisolone group and $2 / 6$ patients in IVIG group. C-reactive protein levels less than $60 \mathrm{mg} / \mathrm{L}$ by day 3 was seen in $17(74 \%)$ in methylprednisolone group and $2(25 \%)$ in IVIG group $(P=0.014)$. There was no mortality. At 2 weeks follow-up coronary artery dilatation persisted in 4 in methylprednisolone group and 1 in IVIG group. Conclusion: In patients with SARS-CoV-2 related MIS-C, methylprednisolone pulse therapy was associated with favorable short-term outcomes.

Keywords: Coronary artery, COVID-19, IVIG, Kawasaki disease.

Published online: April 20, 2021; PII: S097475591600319

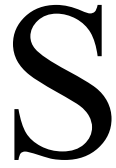
evere acute respiratory syndrome coronavirus 2 (SARS-CoV-2) related multisystem inflammatory syndrome in children (MIS-C) is a dreaded complication that is seen more often in children than in adults [1]. Intravenous immunoglobulin (IVIG) is considered as the treatment of choice for Kawasaki disease (KD) [2]. MIS-C has many dissimilarities with $\mathrm{KD}$, like occurrence in older children (median age 10 years), presence of multi-organ involvement, commonly gastrointestinal tract, myocar-dial dysfunction and shock [3]. MIS-C has been treated empirically with IVIG and steroids [2]. Some studies have used biologicals like tumour necrosis factor inhibitor, interleukin 1 inhibitor, interleukin 6 receptor antibody etc. Most studies have used IVIG alone or in combination with methylprednisolone than methylprednisolone alone in the treatment of MIS-C [1,4]. Nonavailability and high cost of IVIG precludes its use in many centers. Hence, this observational study was conducted to assess the clinical profile and treatment outcome of patients treated with pulse methylprednisolone.

\section{METHODS}

This observational study was conducted in a tertiary care teaching hospital in India. This was the preliminary analysis of an ongoing prospective observational study at the institute. Ethics committee clearance was obtained for the study and informed consent was taken from patient caretakers. Children admitted with MIS-C aged 1 month to 12 years of age from September to November, 2020 were included.

Patients who fulfilled the CDC criteria for diagnosis of MIS-C during the study period were included in the study [5]. Infective causes like dengue, leptospirosis, scrub typhus and bacterial sepsis were excluded by appropriate investigations. SARS-CoV-2 reverse transcriptase polymerase chain reaction (RT-PCR) was done in all patients, and SARS-CoV-2 antibody testing was done using Vitros CoV2T kit [6].

Choice of immunomodulation was decided by the treating unit based on patient demographics and Kerala State guidelines for treatment of children with MIS-C [7]. 
Bedside echocardiography was done in all patients with MIS-C with shock at admission. All patients were subsequently seen by a pediatric cardiologist to look for coronary artery status and cardiac dysfunction. Coronary artery diameter $z$-score $>2$ was considered as coronary artery dilatation/aneurysm [8]. Coronary artery changes like increased echogenicity and non-tapering in the absence of $z$-score $>2$ were taken as nonspecific coronary artery changes. Shock was defined when a patient required more than $20 \mathrm{~mL} / \mathrm{kg}$ of intravenous (IV) fluid resuscitation or inotropic support to maintain blood pressure above the 5 th centile.

Study variables collected using pre-designed proforma included patient demographic characteristics, initial symptoms and clinical signs, laboratory para-meters, type of immunomodulator used, time to deferve-scence, duration of ICU stay, need for inotropic support, duration of shock, duration and type of respiratory support, coronary artery changes at admission and 2 weeks followup and mortality. Patients who were treated with methylprednisolone received pulse dose of $30 \mathrm{mg} / \mathrm{kg}$ once daily for 3 days followed by oral prednisolone at $2 \mathrm{mg} / \mathrm{kg}$ for 1 week or till CRP normalized, whichever was later. Steroid was tapered and stopped over the next 2 - 3 weeks. Children who were treated with IVIG received $2 \mathrm{~g} / \mathrm{kg}$ as a continuous infusion over 8-12 hours with longer duration in patients with cardiac dysfunction.

Time to fever defervescence was recorded at 12-hourly intervals. CRP and D-dimer were repeated on the third and seventh day after the start of IVIG or methylprednisolone. Treatment failure was defined as persistence of fever or worsening of clinical condition beyond 36 hours from the start of first-line therapy or recrudescence of fever within 7 days. Repeat immuno-modulation was considered if fever persisted beyond 36 hours of the first dose of immunomodulatory therapy or if there was a clinical deterioration, irrespective of time since finish of first therapy. Children with treatment failure with IVIG first dose were treated with a second dose of IVIG with pulse methylprednisolone according to the Kerala State guidelines [7]. Children with treatment failure with pulse methylprednisolone were treated with IVIG. All patients were followed up at two weeks after discharge.

All patients with shock were started on low molecular weight heparin (LMWH) at prophylactic dose, which was changed to treatment dose if thrombus was detected. Children on LMWH were transitioned to low dose aspirin once liver enzymes normalized and platelet count increased to more than $80 \times 10^{9} / \mathrm{L}$. Children with thrombus were put on LMWH and anti-platelet dose of aspirin. Anti-inflammatory dose of aspirin $(50 \mathrm{mg} / \mathrm{kg})$ was given in refractory MIS-C with KD like presentation. Children receiving methylprednisolone also received prophylactic IV pantoprazole.

Statistical analysis: Data were entered in MS Excel and analyzed using SPSS 20. Independent sample $t$ test was used for comparison of means. Categorical variables were compared using nonparametric tests. Logistic regression was done to assess the relationship between clinical variables and treatment outcome.

\section{RESULTS}

A total of 32 (males, 21) patients with a median (IQR) age of $7.5(5-9.5)$ years were enrolled. Seventeen patients were antibody positive, 8 patients were both PCR and antibody positive, and two were only PCR positive. Five patients were negative for PCR and antibody but were epidemiologically related to COVID-19 positive cases.

All children presented with fever with a median (IQR) duration of 5 (3-6) days. The clinical characteristics are shown in Table I. The mean (SD) CRP was 141(72) mg/Land ESR 41(33.1) $\mathrm{mm}$ in the first hour. The mean (SD) age of children with shock was significantly higher than those without shock [7.93 (2.27) vs 5.67 (3.39) years; $P=0.02$ ]. Children with shock also had statistically significant higher D dimer [4.75(3.3) vs $1.59(0.982) \mathrm{mcg} / \mathrm{mL} ; P=0.007]$, lower albumin [2.8 (0.40) vs $3.32(0.5) \mathrm{gm} / \mathrm{dL}, P=0.008]$, higher CRP [152 (62.7) vs $120(98.9) \mathrm{mg} / \mathrm{L} ; P=0.049]$, higher lactate [2.35 (1.27) vs $1.01(0.212) \mathrm{mmol} / \mathrm{L} ; P=0.012]$ and lower ejection fraction [53.5 (13.09) vs $65.1(6.29) \% ; P=0.015]$. Eighteen patients $(56 \%)$ had transaminitis but hepatic failure was seen in only one child. Of the four patients with vascular thromboembolic events (VTE), three had thrombus in the left ventricle and one in the right popliteal vein. Even though 10 (31\%) patients were PCR positive, antiviral therapy with remdesivir was offered only to one child in our series.

Table II shows comparative clinical features in children who received pulse methylprednisolone $(n=26)$ or IVIG $(n=6)$. Treatment failure was observed in $2 / 26$ patients in the methylprednisolone group and $2 / 6$ patients in the IVIG group. No child required additional immuno-modulation with immune-biologicals or died during the study period.

Logistic regression was done to assess the effect of clinical variables which were significantly different between the two treatment groups, on the likelihood of occurrence of treatment failure. Logistic regression did not show any effect of age $(P=0.7)$, respiratory support $(P=0.7)$ and five or more organ involvement $(P=0.2)$ on the likelihood of occurrence of treatment failure.

Out of 11 patients with coronary artery dilatation at 
Table I Demographic and Clinical Characteristics of Patients With MIS-C $(N=32)$

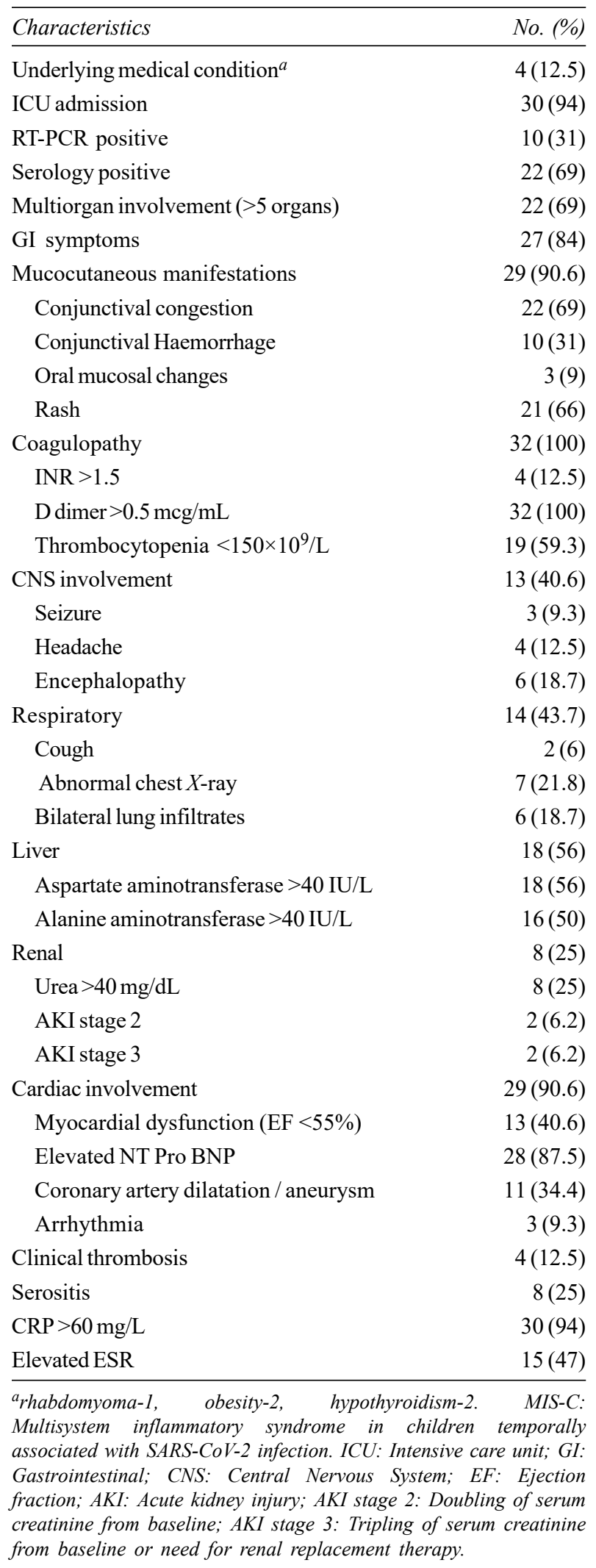

Table II Comparison of Clinical and Outcome Measures in the Two Treatment Groups

\begin{tabular}{|c|c|c|}
\hline Clinical characteristics & $\begin{array}{l}\text { Methyl pre- } \\
\text { dnisolone } \\
\text { group, } n=26\end{array}$ & $\begin{array}{r}I V I G \text { group } \\
n=6\end{array}$ \\
\hline Age, ${ }^{a, e}$ & $8(6-10.25)$ & $3.5(2.4-4.5)$ \\
\hline $\operatorname{ICU}$ stay $(\mathrm{d})^{a}$ & $4.5(3-6.25)$ & $4.5(2-10)$ \\
\hline Hospital stay, (d) ${ }^{a}$ & $11(10-14)$ & $8.5(2.7-21)$ \\
\hline Shock $^{d}$ & $20(77)$ & $2(33)$ \\
\hline Cardiac dysfunction & $11(42)$ & $2(33)$ \\
\hline $\begin{array}{l}\text { Coronary artery dilatation/ } \\
\text { aneurysm }\end{array}$ & $8(30.8)$ & $3(50)$ \\
\hline $\begin{array}{l}\text { Coronary artery non- } \\
\text { specific changes }\end{array}$ & $6(23)$ & 0 \\
\hline$\geq 5$ organ involvement ${ }^{d}$ & $20(77)$ & $2(33)$ \\
\hline Inotropic support & $18(69)$ & $2(33)$ \\
\hline$\geq 2$ inotropes & $14(52)$ & $1(17)$ \\
\hline Respiratory support ${ }^{d}$ & $19(72)$ & $1(17)$ \\
\hline Invasive ventilation & $4(16)$ & 0 \\
\hline CPAP & $14(52)$ & $1(17)$ \\
\hline NIV & $1(4)$ & 0 \\
\hline $\mathrm{CRP}<60 \mathrm{mg} / \mathrm{L}$ on day 3 & $17(68)$ & $2(33)$ \\
\hline D-dimer decrease by day 3 & $19(68)$ & $4(83)$ \\
\hline Repeat immunomodulation & $2(7.7)$ & $2(33.3)$ \\
\hline $\begin{array}{l}\text { Persistent coronary artery } \\
\text { dilatation/aneurysm at } 2 \mathrm{wk}\end{array}$ & $3(12)^{b}$ & $1(25)^{c}$ \\
\hline $\begin{array}{l}\text { Persistent nonspecific coronary } \\
\text { changes }\end{array}$ & $4(16)^{b}$ & $1(25)^{c}$ \\
\hline Normal ejection fraction at $2 \mathrm{wk}$ & $23(92)^{b}$ & $4(100)^{c}$ \\
\hline
\end{tabular}

admission, four had persistent dilatation at two weeks. Six patients $(21 \%)$ had echogenic non-tapering coronaries but coronary artery diameter was less than $2 z$-score. One patient in this group developed coronary dilatation with a $z$-score of more than 2.5 at 2 weeks. LV thrombus had resolved in two patients at 2 weeks follow up while one patient continued to have thrombus at 2 weeks follow up even though the ejection fraction had normalized at 2 weeks. Of the 13 patients with LV dysfunction, 11 (85\%) had normal ejection fraction at 2 weeks follow up. LV systolic function normalized for the remaining 2 patients, at 6 weeks follow up. One child had developed mononeuritis of the right peroneal nerve after one week, which improved with the continuation of steroids and aspirin at antiplatelet dose. 


\section{WHAT THIS STUDY ADDS?}

- Use of pulse methylprednisolone therapy as the first line treatment for MIS-C was associated with favorable immediate and short term follow up outcomes.

\section{DISCUSSION}

The present study reports favorable outcomes in MIS-C with pulse methylprednisolone therapy. MIS-C had dissimilarities to classical $\mathrm{KD}$ like higher age at presentation and higher incidence of GI symptoms and shock, as seen earlier $[3,9,10]$.

Only $6 \%$ of children were referred with a suspected diagnosis of MIS-C, highlighting the fact that MIS-C continues to be a great masquerader. Clinical features in children with acute SARS-CoV-2 infection included fever in $49 \%$, cough in $45 \%$ and GI symptoms in a few [11]. In contrast, all children with MIS-C, had fever with a higher proportion of GI symptoms, while cough was rare $[12,14]$, as also seen in the present study.

As previously reported, more children in our study had conjunctival congestion than oral mucosal changes $[12,13]$; $31 \%$ of children also had conjunctival hemorrhage, which has not been reported in other studies. Breathlessness was also observed in a higher proportion of patients compared to cough [14]. Unilateral lung infiltrates are more frequently reported in acute COVID19 infection in children [15]. While bilateral lung infiltrates were seen in a higher proportion of patients with MIS-C.

A higher seropositivity rate with or without SARSCoV-2 RT-PCR positivity is reported in patients with MIS-C with shock and multiorgan involvement [14]. Presence of positive COVID-19 antibody in patients with positive SARS-CoV-2 PCR at admission probably indicates a greater role of immune-mediated inflam-matory response than acute SARS-CoV-2 viremia in the pathogenesis of MIS-C. As many children with MIS-C have hepatic derangement, use of antiviral therapy in these patients may be counterproductive [13].

Cardiac involvement is the most frequently reported organ dysfunction in MIS-C as also seen in the present study $[1,10,12,6]$. Occurrence of coronary artery aneurysm at follow up in a patient with nonspecific coronary artery changes without dilatation in the initial echocardiogram, highlights the need for meticulous follow up with echocardiogram. Thrombosis has not been reported in similar studies from India $[16,17]$ but reported in studies from the US and UK $[12,13]$.

Earlier studies $[12,14]$ have shown the need for repeat
IVIG and immunomodulators in almost $20 \%$ of those who received IVIG. In our study, only 2 patients who had received steroids subsequently needed IVIG. Logistic regression did not show any relationship between clinical variables like age, shock or multiorgan involvement with initial treatment failure. None of the children required any other alternative immunomodu-lators. There were no deaths or need for ECMO in our study. Earlier studies have reported a mortality of $1.2-2 \%[13,14]$ and need for additional cardiac support with ECMO in $4 \%$ of patients $[12,13]$.

Studies have reported favorable short-term response to IVIG and steroid $[3,14]$. Currently proposed treatment modalities are derived from its similarity with $\mathrm{KD}$ and are based on expert opinion. Treatment with IVIG in resource limited settings is a challenge. In our study, children who received methylprednisolone were significantly older and had a higher number of organ involvement. Outcome measures showed a favorable role for pulse methylprednisolone in the treatment of MIS-C. A recent study [18] also found a more favorable outcome in those treated with IVIG and methylprednisolone than those treated with IVIG alone. Small sample size, observational nature and absence of matched cohorts are the main limitations of the study.

In patients with MIS-C with shock and multi-organ dysfunction syndrome, IV methylprednisolone pulse therapy was associated with favorable immediate and short term follow-up outcomes. Patients with nonspecific coronary changes like absence of tapering and increased echogenicity need to be meticulously followed up for occurrence of coronary artery dilatation even with a low initial $z$-score.

Acknowledgements: Dr S Lakshmi, HOD Pediatric Cardiology, Dr S Bindu, Unit Chiefs, Dr AS Ajith Krishnan, Dr VH Sankar, Dr VK Devakumar and Dr Leela Kumari, SAT, Government Medical College Thiruvananthapuram, who were involved in patient care. Dr K Sarada Devi, HOD, Department of Microbiology, Government Medical College Thiruvananthapuram and Dr Kavitha Raja, Professor, Department of Microbiology, SCTIMST for their support and guidance.

Ethics clearance: Human ethics committee, Medical College, Thiruvnanthapuram; No. 01/32/2021/MCT, dated Jan 15, 2021. Contributors: SS: conceptualized and designed the study, analyzed data and participated in manuscript writing. BS: statistical analysis and interpretation of data, Critical revision of manuscript for intellectual content, GS: Statistical analysis, 
drafting of manuscript, Critical revision of manuscript for intellectual content; NHR: acquisition, analysis and interpretation of data, drafting of manuscript; SKA: supervised the study and contributed to the critical revision of manuscript for intellectual content. All authors approve the final version of manuscript, and are accountable for all aspects related to the study.

Funding: None; Competing interest: None stated.

\section{REFERENCES}

1. Verdoni L, Mazza A, Gervasoni A, et al. An outbreak of severe Kawasaki-like disease at the Italian epicentre of the SARS-CoV-2 epidemic: An observational cohort study. Lancet. 2020;395:1771-8.

2. Jones VG, Mills M, Suarez D, et al. COVID-19 and Kawasaki disease: Novel virus and novel case. Hosp Pediatr. 2020;10:537-40.

3. Jiang L, Tang K, Levin M, et al. COVID-19 and multisystem inflammatory syndrome in children and adolescents. Lancet Infect Dis. 2020;20:e276-88.

4. Abrams JY, Godfred-Cato SE, Oster ME, et al. Multisystem inflammatory syndrome in children associated with severe acute respiratory syndrome Coronavirus 2: A systematic review. J Pediatr. 2020;226:45-54.e1.

5. Centers for Disease Control and Prevention. Multisystem inflammatory syndrome in children (MIS-C) [Internet]. 2020. Accessed February 3, 2021. Available from: https:// www.cdc.gov/mis-c/hcp/

6. Health $\mathrm{C}$ for D and R. EUA Authorized Serology Test Performance. FDA [Internet]. Accessed February 26, 2021. Available from: https://www.fda.gov/ medical-devices/ coronavirus-disease-2019-covid-19-emergency-useauthorizations-medical-devices/eua-authorized-serologytest-performance

7. Government of Kerala. Guidelines-Post-COVID-Clinics. pdf [Internet]. Accessed February 1, 2021. Available from: https://dhs.kerala.gov.in/wp-content/uploads/2020/10/

8. McCrindle BW, Rowley AH, Newburger JW, et al. Diagnosis, Treatment, and Long-term Management of Kawasaki Disease: A Scientific Statement for Health Professionals from the American Heart Association. Circulation. 2017;135:e927.

9. Bhat CS, Gupta L, Balasubramanian S, et al. Hyperinflammatory syndrome in children associated with COVID-19: Need for awareness. Indian Pediatr. 2020;57:929-35.

10. Rowley AH, Shulman ST. The epidemiology and pathogenesis of Kawasaki disease. Front Pediatr [Internet]. 2018. Accessed December 7, 2020. Available from: $h t t p s: / /$ www.ncbi.nlm.nih.gov/pmc/articles/PMC6298241/

11. Meena J, Yadav J, Saini L, et al. Clinical features and outcome of SARS-CoV-2 infection in children: A systematic review and meta-analysis. Indian Pediatr. 2020;57:820-6.

12. Davies $P$, Evans $C$, Kanthimathinathan HK, et al. Intensive care admissions of children with paediatric inflammatory multisystem syndrome temporally associated with SARSCoV-2 (PIMS-TS) in the UK: A multicentre observational study. Lancet Child Adolesc Health. 2020;4:669-77.

13. Feldstein LR, Rose EB, Horwitz SM, et al. Multisystem inflammatory syndrome in US. Children and adolescents. N
Engl J Med. 2020;383:334-46.

14. Godfred-Cato S, Bryant B, Leung J, et al. COVID-19associated multisystem inflammatory syndrome in children - United States, March-July 2020. MMWR Morb Mortal Wkly Rep. 2020;69:1074-80.

15. Kumar J, Meena J, Yadav A, Yadav J. Radiological findings of COVID-19 in children: A systematic review and metaanalysis. J Trop Pediat. 2020:fmaa045.

16. Dhanalakshmi K, Venkatraman A, Balasubramanian S, et al. Epidemiological and clinical profile of pediatric inflammatory multisystem syndrome - temporally associated with SARS-CoV-2 (PIMS-TS) in Indian children. Indian Pediatr. 2020; 57:1010-14.

17. Jain S, Sen S, Lakshmi venkateshiah S, et al. Multisystem inflammatory syndrome in children with COVID-19 in Mumbai, India. Indian Pediatr. 2020;57:1015-9.

18. Ouldali N, Toubiana J, Antona D, et al. Association of intravenous immunoglobulins plus methylprednisolone vs immunoglobulins alone with course of fever in multisystem inflammatory syndrome in children. JAMA.2021;325: 855-64.

19. Harwood R, Allin B, Jones CE, et al. A National Consensus Management Pathway for Paediatric Inflammatory Multisystem Syndrome Temporally Associated With COVID-19 (PIMS-TS): Results of a national Delphi process. Lancet Child Adolesc Health. 2021;5:133-41.

20. Shekerdemian LS, Mahmood NR, Wolfe KK, et al. Characteristics and outcomes of children with coronavirus disease 2019 (COVID-19) infection admitted to US and Canadian pediatric intensive care units. JAMA Pediatr. 2020;174:868-73.

21. Nakra NA, Blumberg DA, Herrera-Guerra A, et al. Multisystem inflammatory syndrome in children (MIS-C) following SARS-CoV-2 infection: Review of clinical presentation, hypothetical pathogenesis, and proposed management. Children (Basel). 2020;7:E69.

22. Riphagen S, Gomez X, Gonzalez-Martinez C, et al. Hyperinflammatory shock in children during COVID-19 pandemic. Lancet. 2020;395:1607-8.

23. McCrindle Brian W, Rowley Anne H, Newburger Jane W, et al. Diagnosis, treatment, and long-term management of Kawasaki disease: A scientific statement for health professionals from the American Heart Association. Circulation. 2017 25;135:e927-99.

24. ToubianaJ, Poirault C, Corsia A, et al. Kawasaki-like multisystem inflammatory syndrome in children during the Covid-19 pandemic in Paris, France: Prospective observational study. BMJ. 2020;369:m2094.

25. Dufort EM, Koumans EH, Chow E J, et al; New York State and Centre for Disease Control and Prevention Multisystem Inflammatory Syndrome in Children Investigation Team. Multisystem inflammatory syndrome in children in New York state. N Engl J Med. 2020;383: 347-58.

26. World Health Organization. Multisystem inflammatory syndrome in children and adolescents temporally related to COVID-19. Accessed August 27, 2020. Available from: https://www.who.int/news-room/commentaries/detail/ multisystem-inflammatory-syndrome-in-children-andadolescents-with-covid-19 